Karolina A. Walkera, Michael L. Unbehauena, Silke B. Lohan, Siavash Saeidpour, Martina C. Meinke, Reinhold Zimmer and Rainer Haag*

\title{
Spin-labeling of Dexamethasone: Radical Stability vs. Temporal Resolution of EPR-Spectroscopy on Biological Samples
}

https://doi.org/10.1515/zpch-2017-1076

Received November 15, 2017; accepted April 5, 2018

Abstract: Spin-labeling active compounds is a convenient way to prepare them for EPR spectroscopy with minimal alteration of the target molecule. In this study we present the labeling reaction of dexamethasone (Dx) with either TEMPO (2,2,6,6-tetramethyl-1-piperidinyloxy) or PCA (3-(carboxy)-2,2,5,5-tetramethyl1-pyrrolidinyloxy) with high yields. According to NMR data, both labels are attached at the primary hydroxy group of the steroid. In subsequent spin-stability measurements both compounds were applied onto HaCaT cells. When the signal of Dx-TEMPO decreased below the detection limit within $3 \mathrm{~h}$, the signal of Dx-PCA remained stable for the same period of time.

Keywords: electron spin relaxation; ex vivo imaging; labeling; X-band EPR.

Dedicated to: Eckart Rühl on the occasion of his 60th birthday.

\section{Introduction}

The functionalization of active drugs with so-called spin labels is relevant for drug delivery. In this field, drugs are embedded in drug delivery devices, e.g.

\footnotetext{
${ }^{a}$ Karolina A. Walker and Michael L. Unbehauen: These authors contributed equally to this work. *Corresponding author: Rainer Haag, Institute for Chemistry and Biochemistry, Freie Universität Berlin, Takustrasse 3, 14195 Berlin, Germany, e-mail: haag@chemie.fu-berlin.de Karolina A. Walker, Michael L. Unbehauen and Reinhold Zimmer: Institute for Chemistry and Biochemistry, Freie Universität Berlin, Takustrasse 3, 14195 Berlin, Germany

Silke B. Lohan and Martina C. Meinke: Department of Dermatology, Venerology and Allergology, Center of Experimental and Applied Cutaneous Physiology, Charité - Universitätsmedizin Berlin, Charitéplatz 1, 10117 Berlin, Germany

Siavash Saeidpour: Department of Physics, Freie Universität Berlin, Arnimallee 14, 14195 Berlin, Germany
} 
nanoparticulate polymeric transporter, providing controlled released out of the polymer matrix $[1,2]$. For the preclinical investigation of a drug delivery device, we need to understand the release mechanism and kinetics of the drug, as well as its fate after being released. Studies on the fate of encapsulated drugs upon release are usually based on UV-Vis or fluorescence spectroscopy techniques [3]. Both techniques require either the use of UV-Vis-active model drugs, such as Nile Red, or fluorescently labeled drugs [4]. Nevertheless, fluorescent labels can significantly change the physico-chemical properties of the drug within the transporter, because they are complex and often large molecules $(M \geq 300 \mathrm{~g} / \mathrm{mol})$ themselves. Using small labels, e.g. spin labels, minimizes the change of properties, such as the drug's polarity, and biological activity [5]. Spin labels, such as nitroxidebased TEMPO and PCA, are sensitive to their chemical microenvironment and hence are used in biological experiments. The difference between TEMPO and PCA in terms of stability of the radical defines their field of application. TEMPO is less stable than PCA and is consequently more readily reduced to the respective hydroxylamine, while reduction of PCA typically occurs under irradiation [6]. The benefit of using spin labels in the field of drug delivery systems is that their sensitivity gives valuable information on the microenvironment of the drug delivery system surrounding the spin label. Combining the spin label with a drug molecule will therefore give us the way to track the movement of the drug inside and outside of the transporter by time-resolved observations [7, 8]. The drug of interest in our case was the poorly water soluble glucocorticoid dexamethasone (Dx, $\log \mathrm{P}=1.83$ ), which is widely used as an anti-inflammatory and immunosuppressant agent $[9,10]$. The drug molecule contains three hydroxy groups that may potentially serve as functional groups for attaching the carboxylic acid-containing nitroxide-based spin labels TEMPO and PCA (see Scheme 1). With respect to the conjugates' further application in drug delivery, it was of great importance to generate only one regioisomer, hence attaching the spin label at selectively one
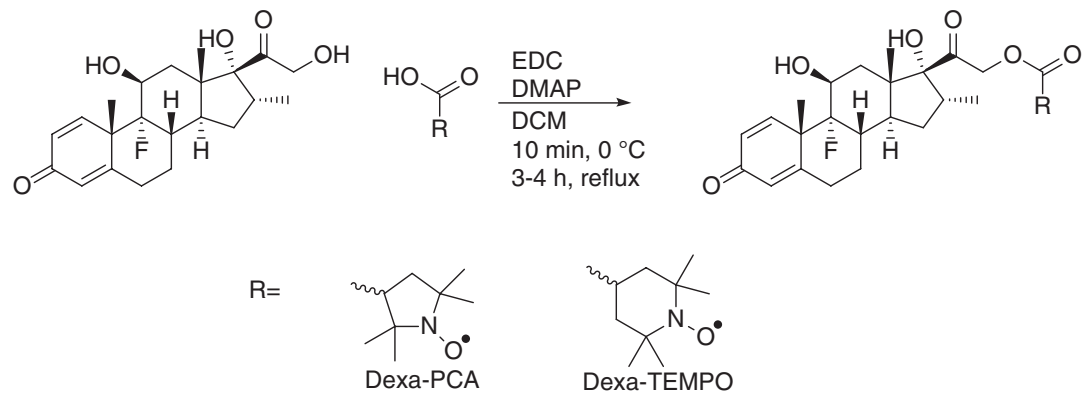

Scheme 1: Synthesis of spin-labeled dexamethasone with either TEMPO or PCA as spin labels. 
hydroxy group of the drug. As a single compound, the spin-labeled drug will have a very defined behavior within the polymeric transporter. We present an easy protocol for the selective ester bond formation between the drug's primary hydroxy group and the spin label, and discuss the differences in terms of EPR performance between the two resulting drug conjugates.

\section{Results}

For the spin-labeling reaction, mild conditions had to be chosen to maintain the spin label's functionality as well as possible. In this regard, the Steglichtype esterification (see Scheme 1) was most promising. This type of reaction involves a carbodiimide, which, in this case, is 1-ethyl-3-(3-dimethylaminopropyl) carbodiimide (EDC) that forms the respective urea scavenging $\mathrm{H}_{2} \mathrm{O}$, the formal side product of this reaction. The acyl-transfer agent 4-dimethylaminopyridine (DMAP) was added to prevent acyl-trapping side products [11-13]. Purification was performed via column chromatography which led to products in high yields of $90 \%$ for Dx-PCA and 88\% for Dx-TEMPO. These yields were significantly higher than previously reported reactions between steroids and spin labels [14].

Dexamethasone exhibits three alcohol groups that can undergo esterification reactions, as depicted in Scheme 1. It was reported that the acyl transfer catalyst DMAP also works for sterically demanding tertiary alcohols [12]. In the case of the three sterically different hydroxy groups of dexamethasone, Steglich-type conditions might potentially lead to the esterification of all three hydroxy groups of the steroid backbone. The conversion and reaction site were determined by mass spectroscopy (ESI-ToF) and NMR, respectively. ESI-ToF spectra only showed peaks that originated from the mono esterification, which indicated that one spin-label had been attached per dexamethasone molecule. In addition to ESI-ToF measurements, the successful binding of the spin label to dexamethasone could be shown by EPR spectroscopy. Figure 1 shows the EPR signal of both the free label TEMPO and the Dx-TEMPO conjugate. A minimization of the high field peak indicates a success in the labeling procedure. Using this spectroscopic technique, we further obtained information about the activity of the labels attached to the drug. Dx-PCA and Dx-TEMPO were compared to freshly prepared solutions of the free spin labels PCA and TEMPO, respectively. Both conjugates showed an EPR activity of $80 \%$ compared to the free drug. This may have been due to quenching by reactive species in solution.

Additionally, NMR analysis was used to determine the site of esterification. Prior to NMR measurements, the unpaired electron of the paramagnetic spin 


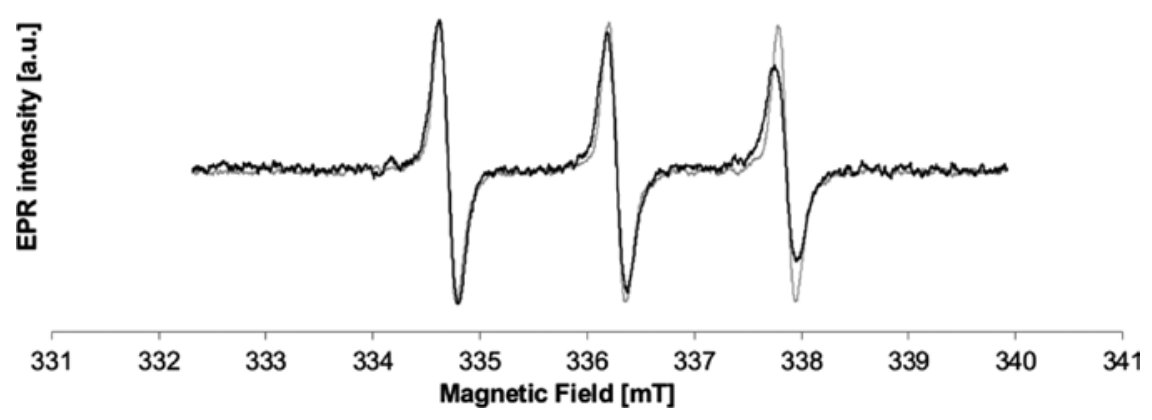

Fig. 1: X-band EPR spectra of TEMPO (gray) and Dx-TEMPO (black) dissolved in DMSO (0.1 mM), both normalized to the maximum height of the low field peak.

label was reduced. The mild reducing agent formic acid was used for the reduction of Dx-TEMPO, while the relatively strong reducing agent zinc powder in combination with ammonium chloride as a proton donor was used for the reduction of Dx-PCA to the respective hydroxylamine (see Scheme 2). Nevertheless, Dx-PCA showed a line broadening which indicates an incomplete reduction of the spin label. Even though the steroidal backbone of Dx-PCA could not be fully resolved, the resulting spectrum gave information on the spin label's binding site.

Based on the spectra in Figure 2, we determined the site of esterification. The essential information is given by the chemical shift of the protons in positions A (one proton) or $\mathrm{C}$ (two protons) that would change upon bond formation. The absence of a shift of peak $\mathrm{A}$ or $\mathrm{C}$ indicates a bond formation at the tertiary hydroxy group at position B. In both the PCA- as well as the TEMPO-labeled conjugates, there was only a significant shift of the two protons of position C. Thus, the coupling reactions performed under these conditions led to an esterification at the primary hydroxy group (C position), which is the sterically preferred position for the spin label attachment $[14,15]$. The tertiary alcohol in the B position features the highest nucleophilicity and thus, by electronic character, best reactivity,<smiles>[R]OC(=O)C1CC(C)(C)N([O])C1(C)C</smiles>
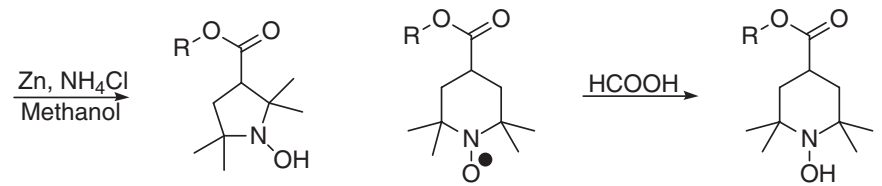

Scheme 2: Reduction of spin-labeled dexamethasone to the respective hydroxylamines. 

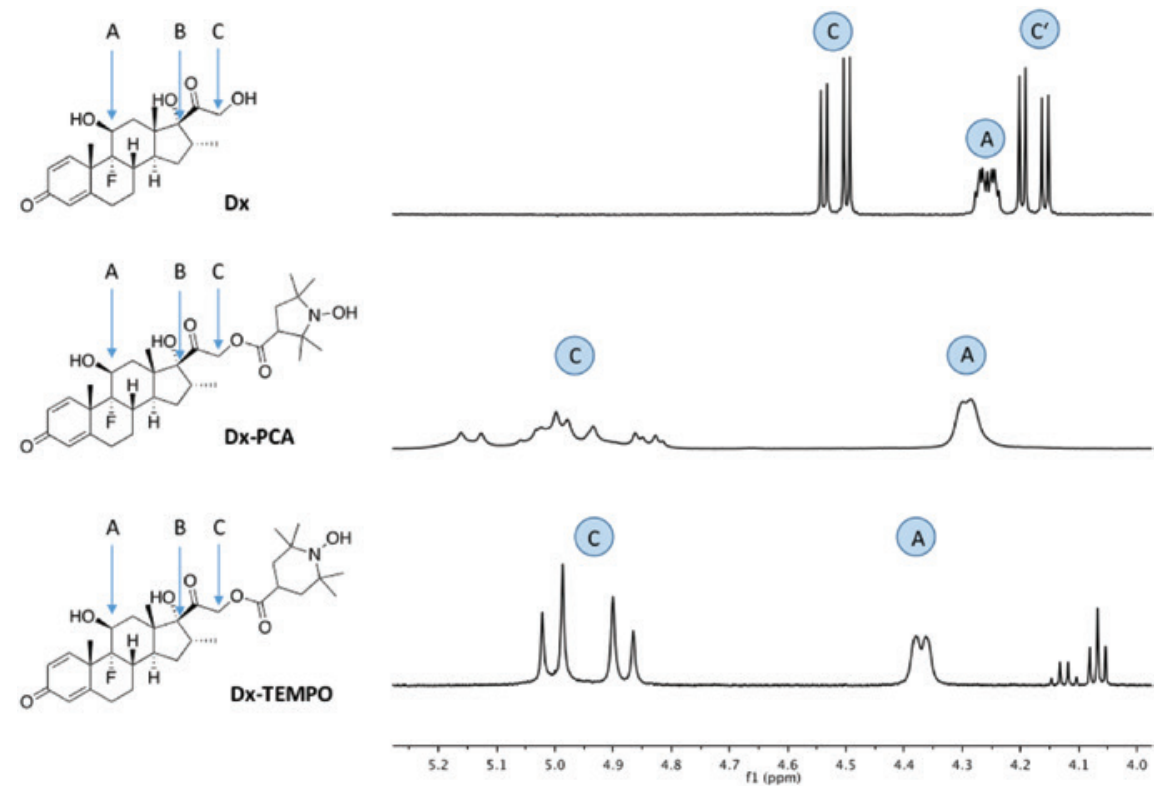

Fig. 2: Section of NMR spectra of Dx, Dx-PCA-NOH, and Dx-TEMPO-NOH (top to bottom) showing peak shift of $\mathrm{CH}^{\mathrm{C}}$, which indicates bond formation to the spin label at position $\mathrm{C}$ of dexamethasone. The strong line broadening in the second spectrum is caused by residual radicals in the sample. Dx-TEMPO: 4.07, $4.14 \mathrm{ppm}$ solvent residues.

followed by the secondary alcohol in position $\mathrm{A}$ and the primary $\mathrm{OH}$-group in position C. By steric demand, the reactivities are reversed, the tertiary alcohol is most hindered and the primary least. The esterification at position $\mathrm{C}$ indicates that our reaction conditions are controlled sterically rather than electronically.

For EPR measurements in ex vivo and cell studies for treatment of skin diseases, the molecular structure of the spin-labeled dexamethasone conjugates had to be stable for a few hours or days after application. We chose a HaCaT keratinocyte cell line as a test model. The stability of labeled conjugates was examined by treating HaCaT keratinocytes with a $0.1 \mathrm{mM}$ Dx-TEMPO or Dx-PCA solution with PBS/glucose solution (2\% DMSO content) for $180 \mathrm{~min}$. PCA and TEMPO showed a stable EPR activity in a control experiment performed in cell medium in the same amount of time (for further details, see the SI). While Dx-TEMPO showed a fast EPR signal decay within 180 min, Dx-PCA showed a stable EPR signal with a marginal decrease to $93 \%$ of the initial EPR signal intensity (see Figure 3). This finding is in good agreement with previously published results reporting a limited stability and increased reactivity of TEMPO in comparison to PCA $[6,16]$. 


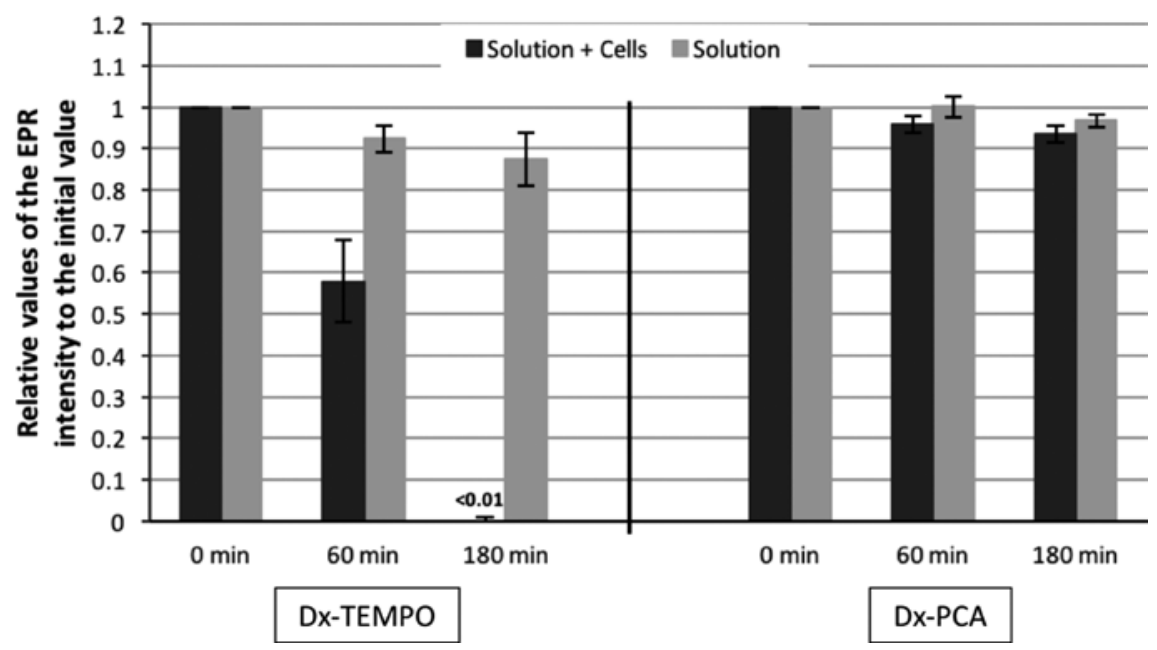

Fig. 3: Investigation of the time-dependent stability of Dx-TEMPO and Dx-PCA (0.1 mM) solution in PBS/glucose (2\% DMSO content) after application to HaCaT cells (black bars). The EPR intensity was measured at different time points ( $0 \mathrm{~min}, 60 \mathrm{~min}, 180 \mathrm{~min}$ ) and compared to the respective conjugates in PBS/glucose (2\% DMSO content, gray bars) without $\mathrm{HaCaT}$ cells. Error bars represent standard errors with $\mathrm{n}=3$.

\section{Conclusions}

The applied protocols for spin-labeling dexamethasone lead to attachment of the spin labels PCA or TEMPO exclusively at the primary hydroxy group of dexamethasone in high yields, which renders the labeling protocol as highly reliable. EPR spectroscopy on HaCaT cells with a focus on the stability of the signal over time revealed that Dx-PCA was more suitable and stable than Dx-TEMPO for tracking the drug pathway over longer periods of time. While the EPR signal of Dx-TEMPO decreased to a non-detectable value within $180 \mathrm{~min}$, Dx-PCA showed a slight decrease to only $93 \%$ of its initial intensity in the same period. We conclude that the more stable Dx-PCA is the conjugate of choice for biological studies on cells above $180 \mathrm{~min}$. If studies are designed for observation periods below $60 \mathrm{~min}, \mathrm{Dx}$-TEMPO will lead to higher temporal resolution and should hence be chosen. EPR spectroscopy does allow not only to track dexamethasone in biological experiments, but also to investigate its chemical environment. Choosing the right stability of spin labels for EPR-based cellular uptake studies can lead to highly temporarily resolved results and give insights on the changing surrounding medium of dexamethasone upon uptake. The presented spin-labeled probe Dx-PCA is especially of high interest for drug delivery research, as it allows to gain deeper insight into the transport mechanisms of new drug delivery systems. 


\section{Experimental section}

\subsection{Synthesis of Dx-PCA}

To a mixture of PCA in dry $\mathrm{CH}_{2} \mathrm{Cl}_{2}$, dexamethasone and DMAP were added under constant stirring. The resulting clear, pale orange solution was cooled to $0{ }^{\circ} \mathrm{C}$ for $10 \mathrm{~min}$ using an ice-bath. EDC was added, stirred for $5 \mathrm{~min}$ at $0^{\circ} \mathrm{C}$ and afterwards allowed to reach r.t. The reaction mixture was refluxed for $4 \mathrm{~h}$. Reaction was monitored using TLC (ethyl acetate). The reaction mixture was extracted with water three times. The collected organic phases were dried with $\mathrm{Na}_{2} \mathrm{SO}_{4}$, yielding a clear yellow solution that was afterwards evaporated to dryness under reduced pressure and redissolved in $\mathrm{CHCl}_{3}$. The crude product was purified using hexane/ethyl acetate (gradient 1:1 to 1:2) as the mobile phase. Yield: $92 \%$. For characterization see SI.

\subsection{Synthesis of Dx-TEMPO}

In an ACE pressure tube, carboxyl-TEMPO (43.5 mg, $0.217 \mathrm{mmol}, 1.3 \mathrm{eq}$ ) was dissolved in dry $\mathrm{CH}_{2} \mathrm{Cl}_{2}(40 \mathrm{~mL})$, followed by the addition of dexamethasone (66.0 mg, $0.166 \mathrm{mmol}$ ) and 4-DMAP (26.4 mg, $0.216 \mathrm{mmol}, 1.3 \mathrm{eq})$ under constant stirring. The resulting clear, pale orange solution was cooled to $0{ }^{\circ} \mathrm{C}$ for $10 \mathrm{~min}$ using an ice-bath. EDC ( $41.7 \mathrm{mg}, 0.218 \mathrm{mmol}, 1.3 \mathrm{eq}$ ) was added, stirred for $5 \mathrm{~min}$ at $0{ }^{\circ} \mathrm{C}$ and afterwards allowed to reach r.t. The reaction mixture was stirred for $3 \mathrm{~h}$ and then heated under reflux. Reaction was monitored using TLC (hexane/ ethyl acetate, 1:1). The reaction mixture was extracted with water three times. The collected organic phases were dried with $\mathrm{Na}_{2} \mathrm{SO}_{4}$, yielding a clear yellow solution which was afterwards evaporated to dryness under reduced pressure and dissolved in $\mathrm{CHCl}_{3}$. The crude product was taken up on silica powder, charged into a chromatography column and purified using hexane/ethyl acetate (gradient 1:1 to 1:2) as the mobile phase. Yield: $88 \%$. For characterization see SI.

\subsection{Cell culture investigations}

HaCaT (human adult low calcium high temperature, secondary keratinocytes) cells were cultivated in RPMI 1640 medium (Gibco, Invitrogen, Carlsbad, CA, USA) with supplements: $1 \%$ penicillin/streptomycin (Biochrom, Berlin, Germany), 2\% glutamine (Biochrom) and 10\% FCS (PAA Laboratories, Vienna, Austria). The cells were cultivated in an incubator at $37^{\circ} \mathrm{C}, 5 \% \mathrm{CO}_{2}$ and $100 \%$ humidity until they reached a confluence of about $80 \%$ which was harvested by trypsination 
(0.5\% trypsin and 0.2\% EDTA, Gibco), counted, seeded in new $75 \mathrm{~cm}^{2}$ flasks, and/ or used for further investigations [17]. To measure the EPR stability of Dx-TEMPO in cells, $1 \times 10^{6}$ cells were incubated in $1 \mathrm{~mL} \mathrm{PBS/glucose} \mathrm{solution} \mathrm{(2 \%} \mathrm{DMSO}$ content) with a $0.1 \mathrm{mM}$ solution of the spin labeled drug in DMSO (final concentration of DMSO $2 \%$ ) at $37{ }^{\circ} \mathrm{C}, 100 \%$ humidity, and $5 \% \mathrm{CO}_{2}$. After incubation, the cells were washed prior to EPR measurements. The baseline EPR intensity was measured by using glass capillaries (Hirschmann Laborgeräte GmbH \& Co. KG, Eberstadt, Germany). TEMPO and PCA spin labels were tested for EPR signal stability in the above-described cell medium, and showed both no signal decay for $180 \mathrm{~min}$. Hence, EPR measurements of TEMPO and PCA conjugates were performed over $180 \mathrm{~min}$. Measurements for $\mathrm{t}=0 \mathrm{~min}$ were started immediately after the application on HaCaT cells. EPR signal intensities represent intracellular spin levels. For further experimental details, see SI.

Acknowledgements: The authors acknowledge support from Deutsche Forschungsgemeinschaft (DFG)/German Research Foundation via SFB 1112, Projects A02 and B01.

\section{References}

1. M. C. Lukowiak, B. N. Thota, R. Haag, Biotechnol. Adv. 33 (2015) 1327.

2. I. N. Kurniasih, J. Keilitz, R. Haag, Chem. Soc. Rev. 44 (2015) 4145.

3. A. Ostrowski, D. Nordmeyer, A. Boreham, C. Holzhausen, L. Mundhenk, C. Graf, M. C. Meinke, A. Vogt, S. Hadam, J. Lademann, E. Rühl, U. Alexiev, A. D. Gruber, Beilstein J. Nanotechnol. 6 (2015) 263.

4. S. Hönzke, C. Gerecke, A. Elpelt, N. Zhang, M. Unbehauen, V. Kral, E. Fleige, F. Paulus, R. Haag, M. Schäfer-Korting, B. Kleuser, S. Hedtrich, J. Control Release 242 (2016) 50.

5. S. Saeidpour, S. B. Lohan, M. Anske, M. Unbehauen, E. Fleige, R. Haag, M. C. Meinke, R. Bittl, C. Teutloff, Eur. J. Pharm. Biopharm. 116 (2017) 94.

6. S. Lohan, A.-C. Lauer, S. Arndt, A. Friedrich, K. Tscherch, S. Haag, M. Darvin, H. Vollert, A. Kleemann, I. Gersonde, N. Groth, J. Lademann, S. Rohn, M. Meinke, Cosmetics 2 (2015) 286.

7. S. F. Haag, E. Fleige, M. Chen, A. Fahr, C. Teutloff, R. Bittl, J. Lademann, M. Schäfer-Korting, R. Haag, M. C. Meinke, Int. J. Pharm. 416 (2011) 223.

8. S. B. Lohan, N. Icken, C. Teutloff, S. Saeidpour, R. Bittl, J. Lademann, E. Fleige, R. Haag, S. F. Haag, M. C. Meinke, Int. J. Pharm. 501 (2016) 271.

9. B. P. Peters, F. G. Weissman, M. A. Gill, Am. J. Health Syst. Pharm. 57 (2000) 645.

10. A. Pyka, M. Babuska, M. Zachariasz, Acta Pol. Pharm. 63 (2006) 159.

11. G. Höfle, W. Steglich, Synthesis 11 (1972) 619.

12. W. Steglich, G. Höfle, Angew. Chem. Int. Ed. Engl. 8 (1969) 981.

13. W. Steglich, B. Neises, Angew. Chem. Int. Ed. Engl. 17 (1978) 522.

14. T. L. Kirley, H. B. Halsall, J. Steroid Biochem. 16 (1982) 133.

15. J. R. Dodd, A. E. Mathew, Steroids 42 (1983) 241. 
16. T. Herrling, J. Fuchs, J. Rehberg, N. Groth, Free Radic. Biol. Med. 35 (2003) 59.

17. S. B. Lohan, S. Bauersachs, S. Ahlberg, N. Baisaeng, C. M. Keck, R. H. Müller, E. Witte, K. Wolk, S. Hackbarth, B. Roder, J. Lademann, M. C. Meinke, Eur. J. Pharm. Biopharm. 89 (2015) 201.

Supplemental Material: The online version of this article offers supplementary material (https:// doi.org/10.1515/zpch-2017-1076). 